Historic, archived document

Do not assume content reflects current scientific knowledge, policies, or practices. 



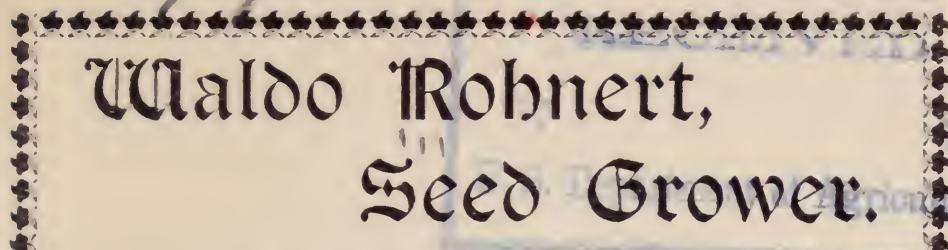

(⿸丆口广

\section{GILROY, CALIFORNIA.}

8

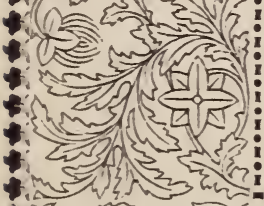

SEED LIST FOR

SEASON 1900

DELIVERY.

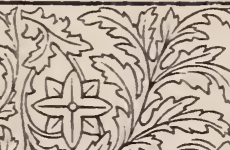

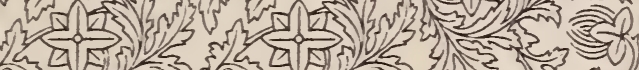

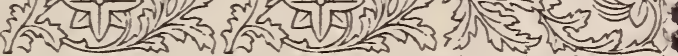

a.

Q.

8

(1)

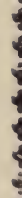

(5)

8

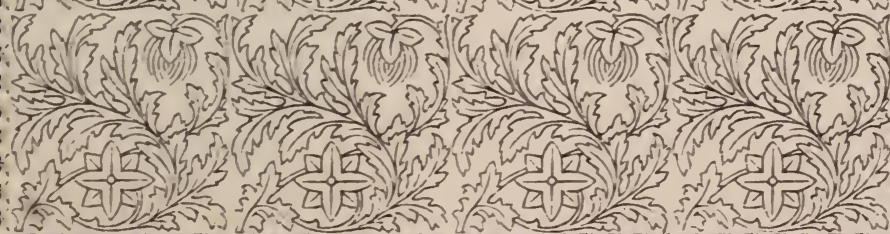

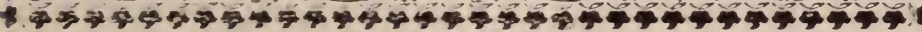





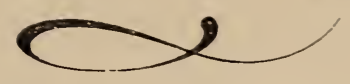

\section{WALDO ROHNERT,}

\section{SEED GROUUER}

\section{Gilroy, California.}

Date

\section{Terms}

While I exercise the greatest care to have all seeds pure and reliable, I do not give any warranty; expressed or implied. If the purchaser does not accept the seeds on these terms and conditions, they must be returned at once, and the money that has been paid for same will be refunded.

IVALDO ROHNERT. 


\section{¿WEET PEAS \%}

QUALITY

PAICE

America.

American Seedlings.

Apple Blossom.

Aurora

Black Knight

Blanche Burpee

Blanche Ferry, Extra Early.

Blushing Beauty.

Burpee's New Countess.

Captain of the Blues.

Captivation.

Celestial.

Chancellor

Colonist

Coquette

Countess of Powis.

Countess of Cadogan

Countess of Radnor.

Crown Jewel.

Cupid Alice Eckford

6 Beauty

66 Primrose 


\section{SWEET PEAS \%}

Cupid Pink..
“ White.
“ Mixed.

Duchess of Sutherland.

Dukc of Sutherland

Duke of Westminster

Dorothy Tennant ...

Earliest of All

Emily Eckford.

Emily Henderson

Golden Gate.

Gorgeous

Gray Friar.

Grandiflora, Mixed.

Her Majesty

Hon. F. Bouverie

Juanita

Katherine Tracy.

Lady Mary Currie.

Lady Nina Balfour.

Lady Penzance.

Lady Skelmersdale 


\section{SWEET PEAS \%}

\section{Lady Grisel Hamilton}

Lottie Hutchins.

Lottie Eckford.

Lovely

Maid of Honor

Mars.

Meteor

Modesty

Monarch.

Mrs. Dugdale

Mírs. Eckford

Mrs. Joseph Chamberlain

Navy Blue.

Oriental.

Othello.

Ovid

Pink Friar

Prima Donna

Prince Edward of York.

Prince of Wales

Queen Victoria.

Ramona 


\section{SWEET PEAS SN}

Red Riding Hood.

Royal Rose...

Sadie Burpee

Salopian

Sensation

Shahzada

Stanley

Stella Morse

Triumph- .

Venus

\section{\% ASTERS, \%}

ROHNERT'S CALIFORNIA NOVELTIES,

Giant Branching Comet Carmine.

66

66

6.6

Carmine,

striped white

Giant Branching Comet, Rose-pink

66

66
66

66
66

66
White.

White,

striped pink 


\section{ASTERS, (Continued.)}

Giant Branching Comet, Mixed

66

66

Upright, White.

Silver Tip, Blue.

66

6" Pink.

Bettridge's Prize Qulled, Mixed.

Comet, Mixed.

Dwarf Crysanthemum, Mixed.

Giant Comet, Mixed.

Giant Comet, White.

Queen of the Market, Mixed

Semples, Crimson

66

Carmine.

66

Lavender.

، Pink

6

Purple

66

White

- 6

Mixed

Perfection, Mixed.

Victoria, Mixed

Washington, Mixed

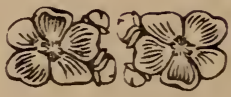




\section{* COSMOS}

QUALITY

Early Dawn.

New Early Blooming.

Mammoth Pink.

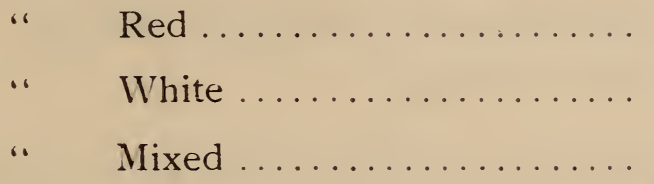

Tints of Dawn

\section{\% MIGNONETTE \%}

Allen's Defiance

Golden Machett.

Pure Machett

Giant Pyramidal

Parsons' White..

Common Sweet.

\section{* VERBENA \%}

Mammoth Auricula-flowered

“ Italian Striped. . . 


\section{VERBENA (Continued.)}

Mammoth Purple.

" Pink ..

"White

Mammoth Fordhook, Mixed

Scarlet Defiance.

\section{ONIONS}

QUALITY

PRICE

- Australian Brown

Yellow Flat Danver

Yellow Globe Danver

Yellow Dutch or Strasburg.

Large Red Wethersfield

Extra Early Red Flat

Prize-taker

Southport Yellow Globe

“ $\quad$ Red “

White Portugal

Philadelphia Silver Skin 

